\title{
Discussion to papers of Sir George Bedbrook, Mr Harris, Dr Klose, Dr Phillippi et al.
}

\author{
Chairman: DR J. Young
}

DR B. A. GreEn (U.S.A.). A combination question on steroids. We did a large study with rhesus monkeys and found that the spinal cord was the feedback pathway for endogenous steroid production as a response to stress. This is well documented in the orthopaedic and general surgical literature. So our rationale of giving high dose steroids over short periods of time is not to make a patient walk again but to allow them to respond to the stresses of the acute period because they are not able to produce endogenous cortisone. There is much in the literature on chronic injury about supplementation with cortisone for abdominal surgery for example. I wonder if in the studies that were reviewed-the cases-the cortisone giving exogenous cortisone decreased the mortality or the morbidity aside from neurologial change.

MR P. HARRIS (G.B.). I would say no, not in our experience.

SIR GEORGE BEDBROoK (Australia). I can definitely answer that question with no; because we tried this for quite a long time with some help from our neurosurgeons, who as you know are very keen on it in cerebral oedema, and we came to the contrary conclusion that in fact we were getting more problems, otherwise we were having more difficulties with our urinary management programmes - that patients were getting secondary problems associated with cortisone. In fact, there were great difficulties because although a rigid programme was laid down, it depends on the weakest member of the chain to effect that programme, and one or two patients were left on cortisone far too long.

MR P. HARRIS (G.B.). I am very worried that some neurosurgeons are using these powerful drugs almost by habit and routinely, and in particular in the case of head injuries. We feel very strongly about this in our department-we are taking in about 1800 head injuries a year-that is a dangerous drug and it doesn't help. We de give them cimetidine routinely as someone else has mentioned, but we are very keen to stop this drug being used in these acute situations.

Sir Ludwig GutTMAnN (G.B.). First of all it is not correct to say that the work at Stoke Mandeville was initially concerned with care, reduction of complications and rehabilitation. It was immediately concerned also with the initial treatment of the injured spine and spinal cord as the most essential part of the comprehensive management of traumatic para- and tetraplegia.

The Miami paper introduced a quantitative method for determining spinal cord function. I am afraid that neurologically this is not a useful quantitative measure for the assessment of spinal cord function, especially as far as sensory function is concerned. I am surprised that the examination, investigation and assessment is left to be done by physiotherapists. Isn't there a doctor who controls this? For testing the motor function you are only repeating, of course, the 40-year-old well-known standard tests of the British Medical Research Council. With regard to sensory assessment, I think it is very unsatisfactory to use, in addition to pain, vibration only as a test for assessing posterior column sensibility. Every neurologist would agree today that vibration is the least reliable sensory test for determining discriminative sensibility. From what we have seen in the slide, the physiotherapist putting the tuning fork on the tummy, on the soft tissue. That is, of course, not correct. I agree with you that one should do sensory tests very carefully and to include all modalities of postural sensibility-that is touch, vibration, joint sense, two-point discrimination and the appreciation of figure writing. If all these tests are done by the doctor who is in charge of the case then you can make comparative quantitative tests. To use only vibration for quantitative assessment must be rejected by 
any physician who is concerned with the clinical, let alone quantitative, examination of spinal cord injuries.

With regard to the paper of the French and Swiss group, this I think was an excellent paper, but I don't think you can compare the results of the operative treatment where you have only 27 and the conservative treatment where you have 270 . Such comparison is not possible. I note that you have given up prostigmine. Of course I saw on your tests that you only inject prostigmine every 8 hours, and I don't think that is satisfactory. If you use prostigmine to treat paralytic ileus with meteorism you have to give it every 3 hours, and before you inject the prostigmine insert a rectal tube and a lot of air is pushed out through the tube. I think you will have better results.

Sir GEORGe BedBrook (Australia). I'd like to ask Dr Klose a question, as to whether it was the same observers each time who recorded the results.

DR KLOSE (U.S.A.). In some instances it was, in others it was not, that is why we did the test reliability to show that from tester to tester there was very little change.

SiR GEORGE BEDBROOK. To those of us who were involved many years ago in polio this was found to be not satisfactory, and that the tests of reliability were in fact suspect; and if you do it on a completely blind nature you find that there is only one real method of doing it and that is that the same observer has to do it every time, because the alteration of technique is so great that it just isn't valid.

DR KLOSE. That's the reason for performing a statistical test reliability. It says that the measure is the same every time no matter who is measuring it.

Dr Green (U.S.A.). With all due respect to Sir Ludwig, the purpose of the study is to create a prospective quantitative way to assess spinal cord function. We are not claiming to do a complete neurological examination. We are claiming to sample each of the major sections of spinal cord anatomically. We found it was difficult to obtain position sense on the belly for example. We found that we were missing large areas of function. What we are hoping to do, and again we are not saying to stop doing a neurological examination because our patients from the time of admission every day receive neurological examinations by neurologists and neurosurgeons but we are creating a research tool to quantitatively determine small changes which we feel the other scales aren't able to do. Position sense and vibration is to sample the dorsal column pain, is to sample the lateral columns and sensation and the motor function, we are trying to eliminate the controversy of $\mathrm{C}_{5} / 6$ in using simple movements like flexion of the elbow and extension. A group of prominent neurologists, neurosurgeons, orthopaedic surgeons and psychiatrists met and they came upon the scale. Now what we are doing is a study; we are not claiming it is a substitute or anything else and we hope that other people use this scale for that purpose. As far as physical therapists: we find them extremely reliable. We only have three that are on 24 hours' duty. We have tested them and their reliability statistically was 0.9 and greater, which was superb, and I would defy any group of physicians to have a better reliability rating in this type of a task. But again this is just a research tool and we are looking at it and we are not making any claims yet, but we are hoping to see small changes. Regarding steroids I agree, we are now part of a nationwide five-centre study to determine the effect of steroids on spinal cord injuries using another scale developed by another centre. We don't know if steroids work, but we are studying it in a prospective statistical fashion so that maybe in 2 or 3 years' time we'll be able to come back and report statistically what we found.

Dr P. MeYer (U.S.A.). I would like to ask Dr Young to comment as the Director of the National Data Centre for Spinal Cord Injuries in the United States on his thoughts concerning the use of the Bardell Index Finder 13 sponsored programmes in the United States. Doctor Harris, I would ask whether you would consider using the word mixed cord rather than sub-total inasmuch as we all have a large number of what you describe as sub-total which are all of the above on your chart, therefore we call it mixed. In reference to the reliability of the Bardell Index, we consider it extremely reliable by multiple observers because the simplicity of the evaluation process has been so narrowed down that the specifics which we are looking for are extremely reliable, depending upon each individual who is doing the evaluation. 
DR Young. Well, I was asked to comment quickly, which I will. When Dr Meyer used the term 'We' it doesn't include me. I don't think it's worth very much at all in spinal cord injuries; perhaps in other neurological disabilities. The use of steroids in the studies we have been able to detect no statistical difference at all in the group with or without steroids. There are some people of course who think perhaps we give ourselves some problems at times with the inadvertent use of them.

Mr P. HarR Is (G.B.). Yes, I think sub-total. I am not happy about the term and I think mixed is a good idea. I'll think about it.

Sir Ludwig Guttmann (G.B.). I have to answer Dr Green. I entirely agree that you should do research, but if you do research you have to accept those tests that are recognised all over the world as physiological tests for testing sensation. You can't just take the vibration for comparative quantitative examination, that would be quite wrong and your research would be useless. Furthermore, some spinal cord patients with sacral sparing. What are you doing with your quantitative tests, in which category do you put them? Please use those recognised physiological parameters on sensation, then you will do an excellent thing.

DR P. Dollfus (France). I want to say that the sacral sparing is something that one must sometimes examine with a glove and do a rectal examination for sacral sparing. Now I don't know if your physiotherapists are capable of doing that or not, but I think that this is of utmost importance. Sometimes we see a very small area of sacral sparing for touch not bigger than an American dime and a few hours later it becomes the size of a dollar and just one sided; and we have also seen a sacral sparing not only in the rectal area but on the penis, which is a very important region, just on one side of the meatus sensation for pinprick not bigger than half a dime, and the patient who was completely recovered and became a D over a few days. So if you don't do the sensory examinations properly I don't think it is of real value. Now the reason why we are not using prostigmine is that we haven't seen any difference between giving prostigmine and giving nothing at all. There are side effects to prostigmine which we do not like, and some side effects on breathing and dryness in the mouth; and we do not use it any longet. A rectal tube is a thing we have always used.

Dr MeYER (U.S.A.). I think it is appropriate to clear up the question which I asked the moderator. Dr Young showed his individual concern over the Bartell Index, but we are talking now about the assessment of neurological recovery and the various methods of attempting to determine whether or not it is resulting from whatever means. On behalf of Dr Guttmann, in an effort to perform a cooperative effort in the United States between I3 Centres: Although we may not represent two countries or several countries we represent 13 different areas and we are selecting the Bartell Index not as the best tool that we can use but a common tool which has been proven statistically to be capable of being performed in 13 different areas and come out with a significant reliability which will allow us commonly to report to the National Data Centre in spite of some built-in bias by the Director of the National Data Centre and, therefore, he will eventually end up giving the report of 13 Centres with his built-in bias, which I think will be very important to all of us. We have found in our Centre that there are two other very subtle changes that probably Dr Guttmann has reported many years ago and I have not had the privilege of reading, or remembering about him, being a student. One is inter-anal proprioception. We have noted that on a number of occasions where perianal sensation is not present a patient will report position sense in the anus, which is as valid as pinprick sensation because it indicates incompleteness. Likewise, we have noted that when a patient who may have a very rapid return of the bulbocavernosus reflex we found it as early as I 5 minutes following complete injury-if you are fortunate enough to receive a patient within I 5 minutes. We have found on several occasions, that the bulbocavernosus returns in that very short period of time. However, the reflexes in the quadriceps and the tendon achilles area do not return so quickly and the presence of reflexes within the early minutes following trauma has turned out to be another sign of incompleteness. It does not demonstrate and it does not indicate that there will be functional sensation or functional return of motor activity, but it has gone along with intra-anal proprioception, 
perianal sensation and early return, very early return, of quadriceps reflex. I see many shaking heads; that's perfectly all right with me, you can shake your head all you like.

DR HAMZa (Egypt). In physiotherapy departments, physical therapy school in Egypt, they like to carry on the examination by themselves; but we oppose this view and, moreover, the functional capabilities which is the function of the occupational therapist. The doctor must assess the patient and make the decisions. I would like to ask Professor Weiss to give us some light about the electrodes which he puts in the spinal cord. 